\title{
ANTIBACTERIAL AND OPTICAL PROPERTIES OF POLYMER OPTICAL FIBERS COATED WITH TITANIUM NANOLAYER USING PLASMA
}

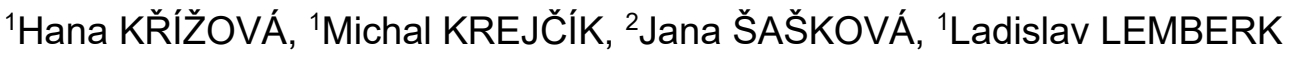 \\ ${ }^{1}$ Technical University of Liberec, Institute for Nanomaterials, Advanced Technologies and Innovation, \\ Department of the Preparation and Analysis of Nanostructures, Liberec, Czech Republic, EU, \\ hana.krizova@tul.cz \\ ${ }^{2}$ Technical University of Liberec, Faculty of Textile Engineering, Department of Material Engineering, Liberec, \\ Czech Republic, EU
}

https://doi.org/10.37904/nanocon.2019.8329

\begin{abstract}
PMMA / PTFE optical fibers with increasing lateral emission were coated with titanium nanolayer using lowtemperature plasma. We investigated how this nanolayer affected the spectrum of emission when VIS LED and UVA LED light sources were used. Even though the FTIR analysis didn't confirm the presence of $\mathrm{TiO}_{2}$ in the titanium nanolayer, the fibers exhibited significantly higher antibacterial properties compared to non-coated fibers. If we don't assume that the titanium surface itself has antibacterial properties, the cause of this phenomenon is in the properties of the formed surface structure. Nevertheless, this treatment could extend the use of polymer optical fibers (POF) to new applications, such as illumination of infectious areas, for example, hospital corridors.
\end{abstract}

Keywords: Polymer optical fibers, titanium nanolayer, low-temperature plasma, antibacterial properties

\section{INTRODUCTION}

An optical fiber is a dielectric waveguide that typically transmits light or infrared radiation along its axis by a process of full internal reflection on the interface of two environments with different refractive indexes. The fiber consists of a core and sheath that provides mechanical protection. It is mostly made of polyethylene, although polyvinyl chloride and chlorinated polyethylene are also used.

\subsection{Polymer optical fibers (POFs)}

Typically, the optical fibers are made from silica, but polymer optical fibers (POFs) from polycarbonate, polymethyl methacrylate, polystyrene or perfluorinated polymers with sensing applications are their cheaper alternative. They have the same benefits as electromagnetic interference resistance, lightweight, and in addition, they are highly flexible. Their disadvantages are higher attenuation (transmission loss), rapid degradation due to prolonged exposure to moisture and high temperatures, their maximum operating temperature is 80 to $100^{\circ} \mathrm{C}$. Then POF loses stiffness and transparency, even if in fact their high-temperature degradation is heavily dependent on the degree of humidity. E.g. if POFs are maintained at $85{ }^{\circ} \mathrm{C}$ with $85 \%$ relative humidity for 1000 hours, the attenuation increases by $0.02 \mathrm{~dB} / \mathrm{m}$. If the relative humidity is about $90 \%$, the attenuation increases by more than $0.03 \mathrm{~dB} / \mathrm{m}$. POFs decompose rapidly at illumination levels of $30-$ $50 \mathrm{~mW} / \mathrm{mm}^{2}$. POF has a tensile modulus of nearly two orders of magnitude lower than that of silicon fiber (2.1 GPa for PMMA and 2.55 GPa for polycarbonate). [1] For this reason, POF with a diameter of $1 \mathrm{~mm}$ is still sufficiently flexible. The minimum bending radius for POFs is less than for classical glass fiber optics.

\subsection{Attenuation}

When the light moves through the optical fiber, it is attenuated (loss of signal transmission) dependent on the light wavelength. Core attenuation increases with their increasing diameter [1]. The optical fiber material has 
a characteristic internal absorption and light scattering, which is a fundamental source of attenuation. The attenuation also occurs due to impurities, defects, and geometric imperfection of the fibers. The attenuation of silica fibers decreases with increasing wavelength. Their lowest attenuation lies in the near infrared range of $1300-1600 \mathrm{~nm}$, which is the area of wavelength range typically operated by silica optical fibers. POFs have the lowest attenuation in the visible light area, therefore, they are advantageously used to transmit the light signal in the range of $400-700 \mathrm{~nm}$. [2] In general, the attenuation of POFs is greater than the attenuation of silica optic fibers and they lead a signal effectively to only a few hundred meters away. Therefore, POFs are used for data transmission over short distances, such as home internet, automotive applications [3], and for biomedical applications [4].

\subsection{POFs with lateral emission}

For a variety of applications (e.g. active safety textiles), it is necessary to allow lateral radiation of optical fibers. The lateral emission can be achieved if the angle of incident light is less than the critical angle. This can be achieved either by increasing the refractive index of the sheath, or by decreasing the refractive index of the core, or by changing the angle of incident light.

It is possible to use multiple micro-bending of the core or sheath, dispersing additives, or fluorescence, either to the core or the sheath or to create geometric asymmetry in the core/sheath system. Laterally emitting optical fibers can compete with light emitting diodes (LED) in the future, especially if controlled local radiation is easy to achieve. Optical fibers with lateral emission can be incorporated into a wide range of textile structures such as labels, tapes, strips, cords, woven and knitted fabrics. They found applications as light-signaling components of clothing (cycling jackets, backpacks, work jackets for policemen, rescuers, etc.), textile light panels, textile displays, materials for illumination, etc. [5]

\subsection{Low - temperature plasma technology}

Low-temperature plasma technology is a suitable method for preparation of thin film or thin layers by physical sputtering. Plasma can be created in the laboratory by applying direct or alternating high voltage to a gas. Using the gas at reduced pressure (partial vacuum), one can thereby obtain either a low-temperature, nonequilibrium "glow discharge" type of plasma. [6] With the supplied energy, collision processes among the constituent particles become violent enough for these to break apart. This results in the formation of number densities of charge-bearing sub-particles, namely electrons and ions. These highly energetic electrons are responsible for initiating chemical reactions, by breaking covalent chemical bonds of ground-state gas molecules in the course of collision-induced energy transfer. The gas molecules and ions, the "heavy" particles, can be kept "cold" (near ambient temperature, $300 \mathrm{~K}$ ), while the electron gas can be made very "hot", well above $10^{4} \mathrm{~K}$. Through the selection of gas or gas mixture, method of energy input, and reactor geometry, the conditions for a very wide range of technological process applications can be optimized.

\subsection{Objectives of this study}

In this study, we tried to extend the practical application of POF's by plasma modification of their surface to achieve a surface unfavorable for bacterial settling and multiplication or biofilm formation, while maintaining acceptable luminous intensity.

\section{EXPERIMENTAL PART}

\subsection{Experiment procedure in brief}

Coating of polymer optic fiber with titanium nanolayer using low-temperature plasma in the presence of lowpressure oxygen-argon atmosphere. Immersion of coated (POF/Ti) and non-coated (POF/0) fibers in bacterial 
suspension ( $\mathrm{G}+$ bacteria separated from the skin smear) for 2 minutes. Connecting of both fibers to light sources (UVA LED or VIS LED $6500 \mathrm{~K}$ ) for 0-30-60 minutes. Imprinting of the fiber surfaces into the agar medium. Cultivation $\left(24 \mathrm{~h}, 37^{\circ} \mathrm{C}\right)$ and evaluation of the differences in the cultured bacterial colonies from both fibers.

\subsection{Material}

Polymer optical fiber Grace GSPOF-300R with increased lateral emission, diameter $3.0 \mathrm{~mm}$ (Grace Plastic Optical Fiber Co. Ltd., TW)

TSB liquid culture medium and TSA solid culture medium in Petri dishes (Oxoid, CZ)

\subsection{Devices}

Laboratory plasma chamber 120 liters (PL), plasma generator Cesar RF (13,54 MHz/kW), Advanced Energy (US), magnetron sputtering TORUS, KJLC® (US)

UV LED power light source PROLIGHT OPTO PM2L-3LLS-SD with emission spectrum from 390 to $410 \mathrm{~nm}$ $\left(\lambda_{\max } 400 \mathrm{~nm}\right.$ ), with LED current source $680 \mathrm{~mA}$ for 1-14 LEDs (LED dimming dim driver LEDISCON (CZ), type LDT 150-068)

VIS LED $3 \mathrm{~W}, 6500 \mathrm{~K}$ light source, with 400-650 nm emission spectrum, ( $\left.\lambda 1_{\max } 450, \lambda 2 \max 550 \mathrm{~nm}\right)$

FTIR: ATR technique on Ge crystal, Nicolet ${ }^{\mathrm{TM}}$ iS10 FTIR Spectrometer Thermo Scientific ${ }^{\mathrm{TM}}$ (US)

Surface roughness analysis of fibers: Mechanical profilometer Bruker Dektak XT (US)

Lateral emission and light output of fibers: device POFIN1 (CZ) with sensor THORLABS PM 1000 SB (JP)

\subsection{Methods used}

\subsubsection{Preparation and analysis of titanium nanolayer on the POF surface}

A $15 \mathrm{~cm}$ long POF was placed in the plasma chamber. The coating of the fiber using a titanium target was carried out under the following conditions: bias in plasma $120 \mathrm{~V}$, magnetron power $800 \mathrm{~W}$, plasma time 12 minutes, pressure in the plasma chamber $0.6 \mathrm{~Pa}$, composition of the atmosphere in the plasma chamber: $\mathrm{Ar}$ $12 \mathrm{sccm},\left(\mathrm{sccm}=\right.$ standard cubic centimeters or $\mathrm{ml} / \mathrm{min}$ under standard conditions), $\mathrm{O}_{2} 7 \mathrm{sccm}$. The POF/Ti and POF/0 surfaces were analyzed by FTIR spectroscopy (mechanical separation was first performed on POF/0 to identify the sheath and core composition). Furthermore, the surface roughness analysis of both fibers was performed using a profilometer. The intensity of POF/Ti and POF/O lateral emission was measured according to the internal TUL standard. [7]

\subsubsection{Antibacterial tests}

To test the antibacterial properties of the POF surface by UV LED, a bacterial suspension of randomly selected and cultured $\mathrm{G}+$ bacterial strain was prepared from a pre-cultured skin smear microflora. This bacterial suspension of selected and in TSB cultured strain was diluted using sterilized spring water to a concentration of $10^{5} \mathrm{CFU} / \mathrm{ml}$. The suspension was used to inoculate the $15 \mathrm{~cm}$ long fiber. Respectively, one end of the fiber was attached to an LED power source, the free end of the fiber was wetted in $50 \mathrm{ml}$ of bacterial suspension for 2 minutes at room temperature. After removal from the bacterial suspension, the source / fiber assembly was mounted in an upright position by the inoculated and slightly curved end of the flexible POF upward. The system was placed in the dark at $18{ }^{\circ} \mathrm{C}$ without airflow and left for 30 minutes without power on to allow dripping and partially drying of adhering drops of the bacterial suspension. Thereafter, the first POF-end imprint was transferred to the agar on Petri dish (TSA). This was followed by rinsing under a stream of cold sterilized spring water, sterile drying and re-immersion in the bacterial suspension for 2 minutes. Subsequently, the entire assembly was again left in the dark for 30 minutes. Then the UV LED light source was plugged in to set the dimmable driver to its maximum. After 30 minutes, another POF end print was made, followed by rinsing, 
drying, new inoculation, $30 \mathrm{~min}$ in the dark, and then the UV LED source was turned on for 1 hour. Subsequently, the inoculated end of the fiber was imprinted on the agar. The same procedure was performed with POF/Ti and POF/0. All cultivations of bacteria were run at $37^{\circ} \mathrm{C}$ for 24 hours.

\section{RESULTS AND DISCUSSION}

As shown in Figure 1 and Figure 2, the POF type used was composed of PMMA (core) and poly (tetrafluoroethylene) (sheath). FTIR analysis of the coated POF/Ti surface despite the presence of oxygen in the plasma chamber atmosphere did not show the presence of $\mathrm{TiO}_{2}$, only the presence of pure titanium.

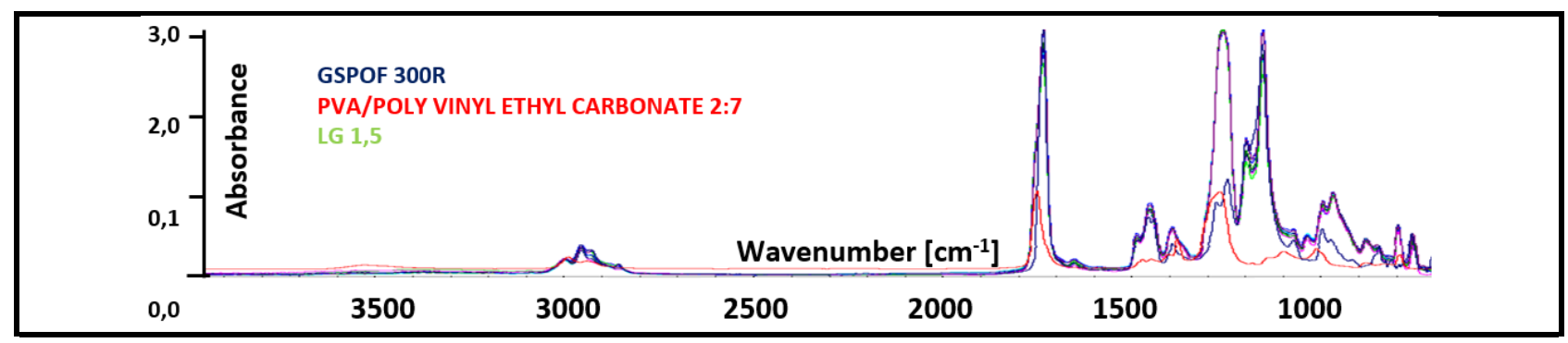

Figure 1 FTIR spectra of GSPOF-300R core: Acrylic (probably PMMA)

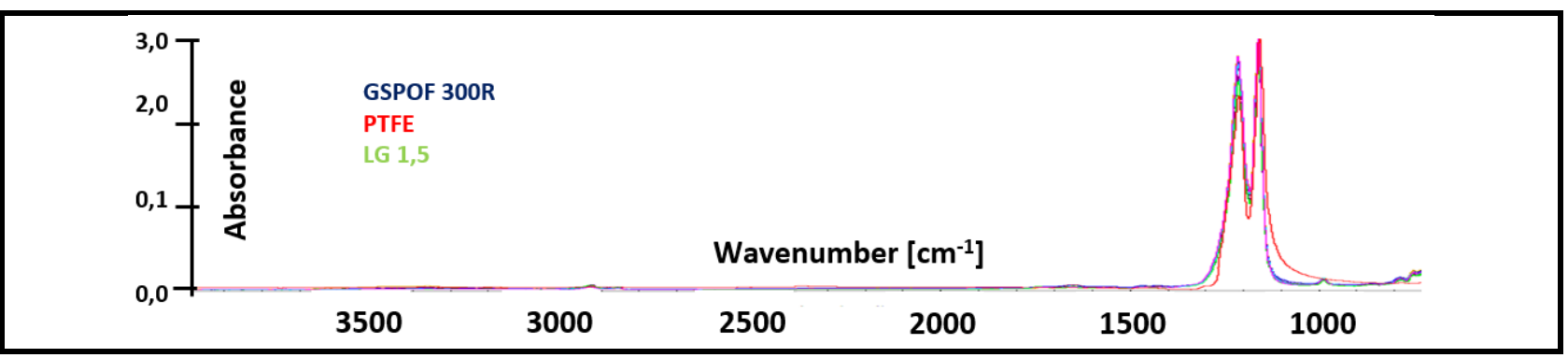

Figure 2 FTIR spectra of GSPOF-300R sheath: poly (tetrafluoroethylene)

Figures 3 and 4 show the surface of the POF/Ti and POF/O. Although the absolute difference between the lowest and the highest point of the scanned surface (Rt parameter) is similar for both fibers $(6.681 \mu \mathrm{m}$ for $\mathrm{POF} / \mathrm{Ti}$ and $5.937 \mu \mathrm{m}$ for POF/0), and although the POF/Ti is more rugged, in fact, its roughness is lower than the surface of untreated fiber POF/0. It results from the Ra values, which are the average height of unevenness in the surface: $\mathrm{Ra}(\mathrm{POF} / \mathrm{Ti}$ ) was $0.354, \mathrm{Ra}(\mathrm{POF} / 0)$ was 0.515 . Lower POF/Ti surface roughness could be the reason why bacteria may not be able to keep on its surface. The results of the bacterial test are shown in Figure 5 and Figure 6. Figure 5 shows the apparent loss of bacteria in the POF/Ti imprint in the TSA at intervals of $0-30-60$ minutes of connection to the UV LED source. If UVA radiation is the cause of their significant decreasing, the same significant decreasing of bacteria would also occur with untreated POF/0 fiber (Figure 6). However, this was not confirmed, on the contrary, there was a stronger increase of CFUs on POF/0. Figure 7 shows the difference in the quantity and size of CFUs cultivated from imprints of inoculated fiber ends. Both fibers were attached to the same VIS LED source for 6 hours: no disinfection occurred on POF/0 due to visible light radiation. In addition, as shown by the POF/Ti spectrum measured by POFIN1 device (Figure 8), the titanium nanolayer on the fiber surface caused UV attenuation: lateral emission of POF/Ti was about a quarter compared to POF/0 when using UV LED. Since the increase in the thickness of the plasma titanium layer is substantially linear over time (using this power and plasma setting, it is approximately 10 $\mathrm{nm} / \mathrm{min}$, Figure 9), the predicted titanium layer thickness was $120 \mathrm{~nm}$. Therefore, it is desirable to prepare a thinner layer of titanium on the POF surface. It means to reduce the plasma time when setting the plasma conditions given in section 2.4.1. 


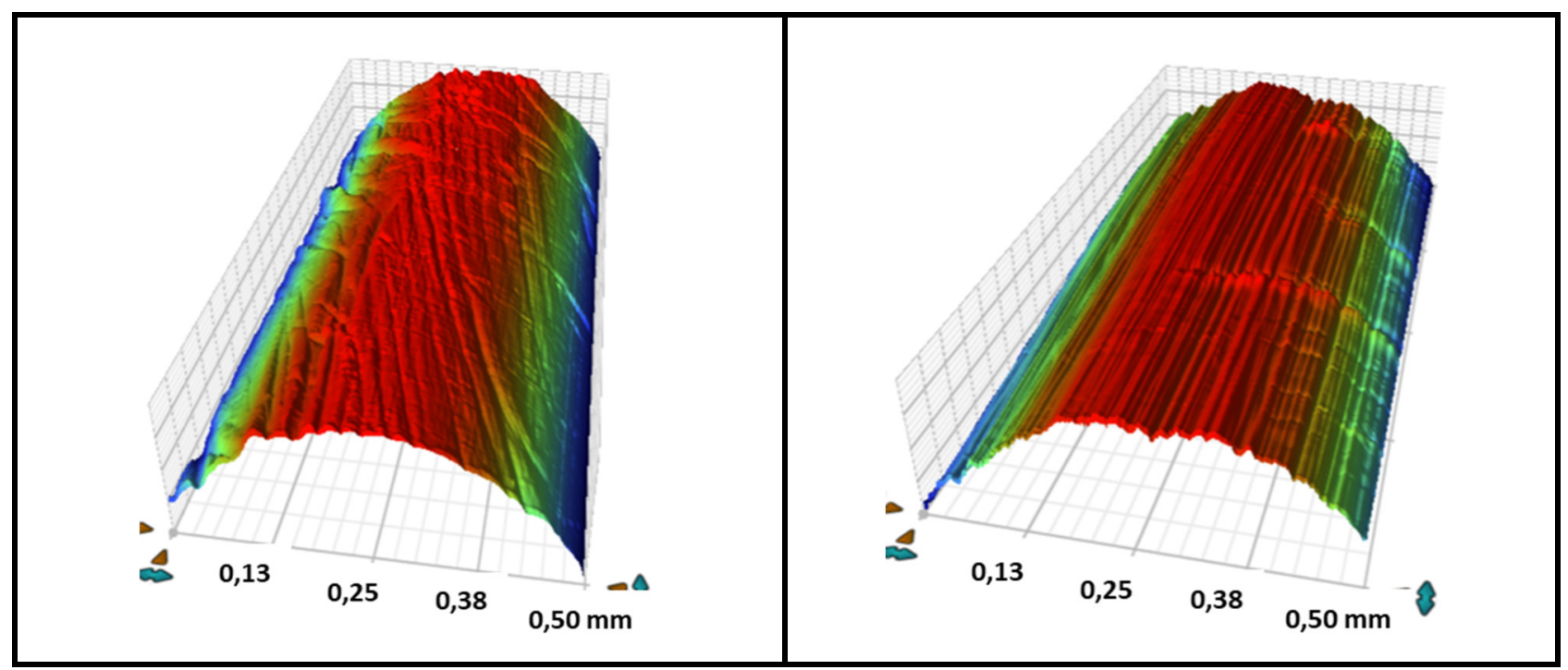

Figure 3 Analysis of POF/Ti surface roughness

Figure 4 Analysis of POF/0 surface roughness

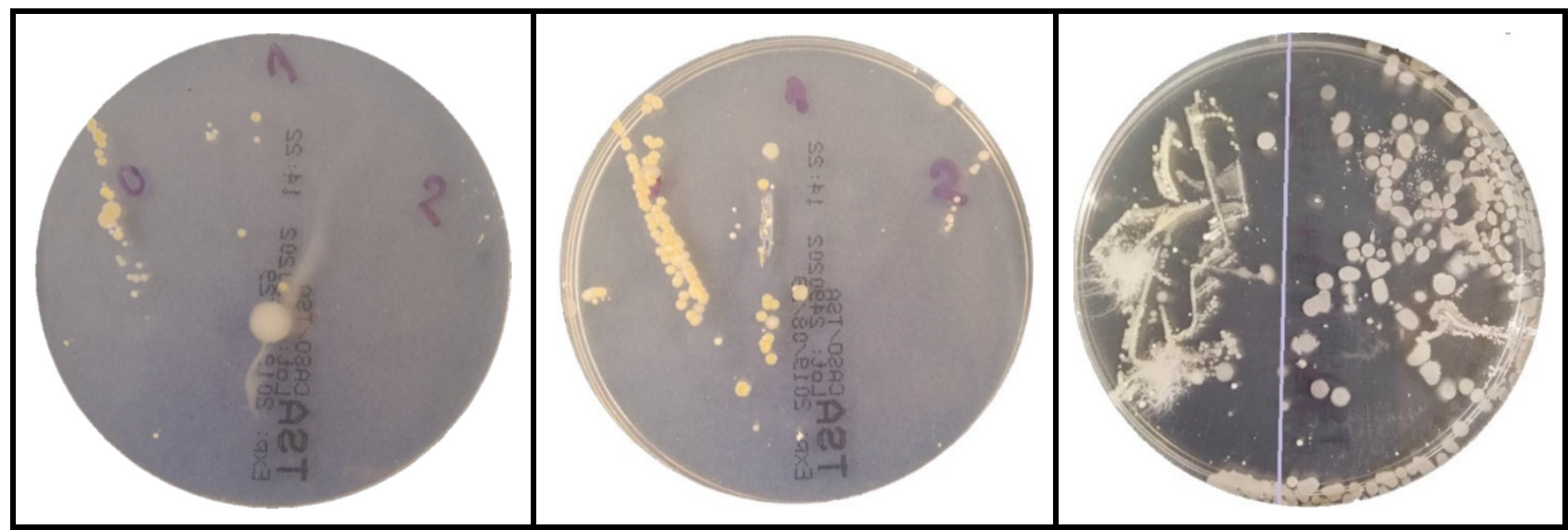

Figure 5 CFU cultivated from imprints of inoculated POF/Ti after $0-30-60$ minutes of UVA
Figure 6 CFU cultivated from imprints of inoculated POF/0 after 0 - 30 - 60 minutes of UVA
Figure $7 \mathrm{CFU}$ cultivated from an imprint of inoculated POF/Ti (left) and POF/0 (right) after 6 hours of VIS light

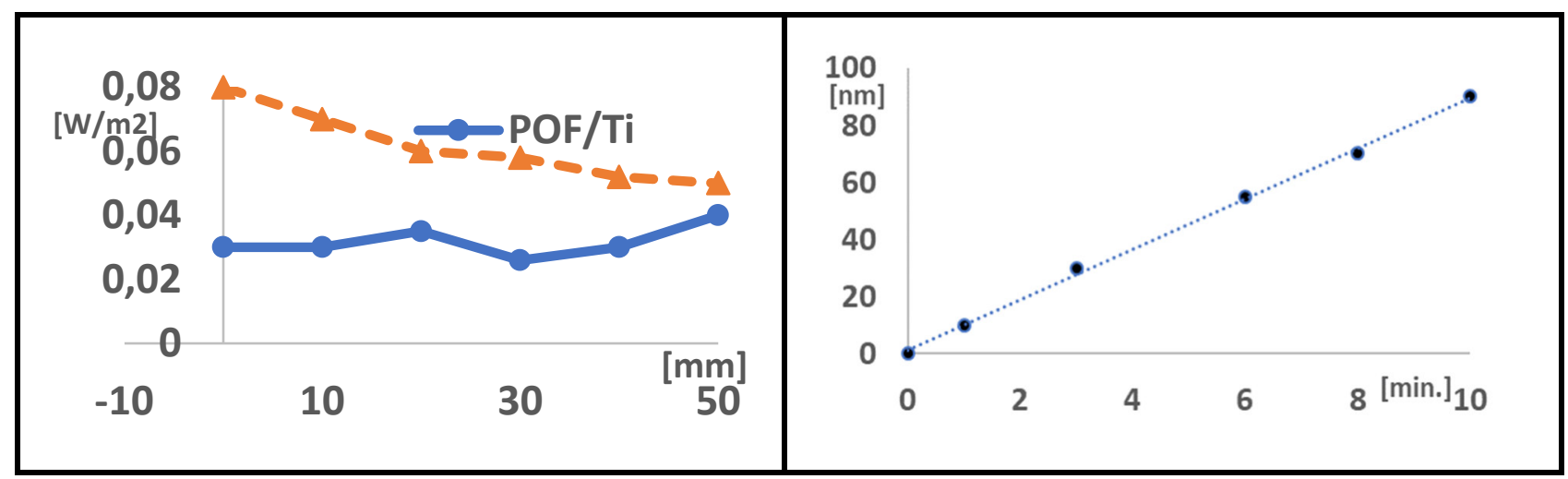

Figure 8 Lateral emission of both fibers using UVA LED
Figure 9 The thickness of the plasma titanium layer (on a glass surface) as a function of the time 


\section{CONCLUSION}

PMMA/PTFE optical fiber was coated with titanium in an $\mathrm{Ar} / \mathrm{O}_{2}$ atmosphere using low-temperature plasma. The fiber according to FTIR analysis contain pure titanium. The layer was $120 \mathrm{~nm}$ thick. It decreased the luminous output of the lateral emission to a quarter when the UVA LED was connected. Nevertheless, this fiber has been shown to have improved antibacterial properties compared to POF without plasma treatment. Reduced bacterial growth was evident even when using VIS LED light for 6 hours. This was probably due to the less roughness of the surface compared to the non-coated POF, therefore, a smaller number of bacteria from the inoculum suspension were captured. Plasma sputtering time of 12 minutes has proven not to be quite suitable since the titanium coating layer formed was $120 \mathrm{~nm}$ thick. This reduced the output of the laterally emitted light to a quarter. The shorter plasma time with the formation of a thinner layer should not affect the surface properties but will allow a higher fiber luminance. Although the proposed modification exhibited only partial antibacterial properties, the POF thus modified is in principle suitable for illuminating areas exposed to infectious agents. In the long run, it could prevent the formation of a bacterial biofilm using UVA or VIS light sources. It could be used in long-term and economical lighting of hospital corridors.

\section{ACKNOWLEDGEMENTS}

This study is a result of the research funded by the project TAČR EPSILON - TH04020405

\section{REFERENCES}

[1] KŘEMENÁKOVÁ, D., MILITKÝ, J., ŠESTÁK, J. Vlákenné struktury pro speciální aplikace. 1st ed. Kanina: 2013.

[2] PETERS, K. Polymer optical fiber sensors-a review. Smart materials and structures. 2010, vol. 20, no. 1, p. $13002-13017$.

[3] Application of Polymer Optical and Glass Fibers. In: ZIEMAN, O., et al. POF Handbook. Berlin, Heidelberg: Springer, 2008, pp. 593-663.

[4] CENNAMO, N., et al. An innovative plastic optical fiber-based biosensor for new bio/applications. The case of celiac disease. Sensors and Actuators B: Chemical. 2013, vol. 176, pp. 1008-1014.

[5] HASHIMOTO, S., et al. LightCloth: senseable illuminating optical fiber cloth for creating interactive surfaces. In SIGCHI Conference on Human Factors in Computing Systems. Paris: 2013, pp. 603-606.

[6] D'AGOSTINO, R., et al. Low-temperature plasma processing of materials: past, present, and future. Plasma Processes and Polymers. 2005, vol. 2, no. 1, pp. 7-15.

[7] Internal standard IN. 21-601-01/01. Evaluation of emission intensity of optical fibers, fiber bundles and textiles with embedded optical fibers. TUL, 2013. 